\title{
hnRNPK-regulated PTOV1-AS1 modulates heme oxygenase-1 expression via miR-1207-5p
}

\author{
Chang Hoon Shin ${ }^{1}$, Seongho Ryu ${ }^{2}$ \& Hyeon Ho Kim ${ }^{1,3, *}$ \\ ${ }^{1}$ Department of Health Sciences and Technology, Samsung Advanced Institute for Health Sciences and Technology, Sungkyunkwan \\ University, Seoul 06351, ${ }^{2}$ Soonchunhyang Institute of Medi-bio Science (SIMS), Soonchunhyang University, Cheonan 31151 , ${ }^{3}$ Institute for \\ Future Medicine, Samsung Medical Center, Seoul 06351, Korea
}

\begin{abstract}
Antisense transcripts were initially identified as transcriptional noise, but have since been reported to play an important role in the quality control of miRNA functions. In this report, we tested the hypothesis that heterogeneous nuclear ribonucleoprotein $\mathrm{K}$ (hnRNPK) regulates miRNA function via competitive endogenous RNAs, such as pseudogenes, long non-coding RNAs, and antisense transcripts. Based on analyses of RNA sequencing data, the knockdown of hnRNPK decreased the antisense PTOV1-AS1 transcript which harbors five binding sites for miR-1207-5p. We identified heme oxygenase-1 (HO-1) mRNA as a novel target of miR-1207-5p by western blotting and Ago2 immunoprecipitation. The knockdown of hnRNPK or PTOV1-AS1 suppressed HO-1 expression by increasing the enrichment of $\mathrm{HO}-1$ mRNA in miR-1207-5p-mediated miRISC. Downregulation of HO-1 by a miR-1207-5p mimic or knockdown of hnRNPK and PTOV1AS1 inhibited the proliferation and clonogenic ability of HeLa cells. Taken together, our results demonstrate that hnRNPKregulated PTOV1-AS1 modulates HO-1 expression via miR1207-5p. [BMB Reports 2017; 50(4): 220-225]
\end{abstract}

\section{INTRODUCTION}

Approximately $40 \%$ of annotated transcripts in humans are transcribed in both directions, indicating that antisense transcription occurs at a significant proportion of the human genome $(1,2)$. Antisense transcripts were initially classified as transcriptional noise (3). However, they have recently been identified as functional non-coding RNAs and influence almost all steps of gene regulation, including transcriptional and post-transcriptional gene regulation. For tight control of gene

*Corresponding author. Tel: +82-2-3410-1039; Fax: +82-2-34100534; E-mail: hyeonhkim@skku.edu

https://doi.org/10.5483/BMBRep.2017.50.4.024

Received 9 February 2017, Revised 13 February 2017, Accepted 22 February 2017

Keywords: hnRNPK, HO-1, miR-1207-5p, PTOV1-AS1 expression, antisense transcripts compete with microRNAs (miRNAs) for binding to target mRNA and thus act as miRNA sponges.

The miRNAs, which are small non-coding RNAs, generally suppress the expression of target genes by binding to partially complementary sequences (miRNA response elements, MREs) in the 3' untranslated region (UTR) of target mRNA (4). The activity of miRNAs can be affected by competitive endogenous RNAs (ceRNAs) which harbor MREs $(5,6)$. ceRNAs play a regulatory role in the action of single or multiple miRNAs by influencing the accessibility of miRNAs in target mRNAs. Therefore, they act as miRNA quantity controllers that govern the expression level of miRNAs, influencing the suppression of target mRNAs.

Heme oxygenase-1 (HO-1, encoded by HMOX1) is an inducible liver microsomal protein and plays an important role in the cytoprotective machinery. HO-1 is commonly induced by cellular stresses, including heavy metals, endotoxin, hypoxia, nitric oxide, and ultraviolet irradiation (7). In cancer cells, HO-1 is highly expressed and upregulated by anti-cancer agents, making cancer cells resistant to treatment (8). Overexpression of HO-1 also confers resistance to oxidative stress and increases angiogenic potential (9).

Since heterogeneous nuclear ribonucleoprotein K (hnRNPK) plays a critical role in the proliferation and metastasis of various cancer cells, we searched for hnRNPK-regulated antisense transcripts and their target miRNAs. We determined that hnRNPK positively regulates the level of prostate tumor overexpressed 1-antisense 1 (PTOV1-AS1) which harbors five binding sites for miR-1207-5p. The knockdown of hnRNPK or PTOV1-AS1 increased the enrichment of HO-1 mRNA in miR-1207-5p-mediated miRNA-induced silencing complex (miRISC) and thus suppressed the expression of HO-1. Decreased HO-1 expression by a miR-1207-5p mimic or PTOV1-AS1 siRNA inhibited proliferation and clonogenic ability.

\section{RESULTS}

Knockdown of hnRNPK decreases PTOV1-AS1 expression We found that significant numbers of antisense transcripts

ISSN: 1976-670X (electronic edition)

Copyright (C) 2017 by the The Korean Society for Biochemistry and Molecular Biology

(c) This is an open-access article distributed under the terms of the Creative Commons Attribution Non-Commercial License (http://creativecommons.org/licenses/by-nc/4.0) which permits unrestricted non-commercial use, distribution, and reproduction in any medium, provided the original work is properly cited. 
were regulated by hnRNPK, including EMC3-AS1, FLVCR1AS1, VIM-AS1, ZBED5-AS1, through our efforts to search for hnRNPK-regulated long non-coding RNAs (IncRNAs). Of these transcripts, we focused on the functions of PTOV1-AS1 in this study because its sense transcript PTOV1 is known to function as an oncogene. To validate the effect of hnRNPK on PTOV1AS1 expression, HeLa cells were transfected with control or hnRNPK siRNA. Cells transfected with specific hnRNPK siRNA showed an efficient decrease of hnRNPK (Fig. 1A). The level of PTOV1-AS1 was assessed by RT-qPCR and semi-quantitative PCR (Fig. 1B). As expected, hnRNPK-silenced cells showed decreased expression of PTOV1-AS1. Cellular fractionation was performed to examine the localization of PTOV1-AS1. The levels of $\alpha$-tubulin and lamin B were checked to confirm cytoplasmic and nuclear fractions (Fig. 1C, left panel). We found that PTOV1-AS1 was preferentially located in the cytoplasm and therefore speculated that it might function with posttranscriptional gene regulators such as miRNAs (Fig. 1C,
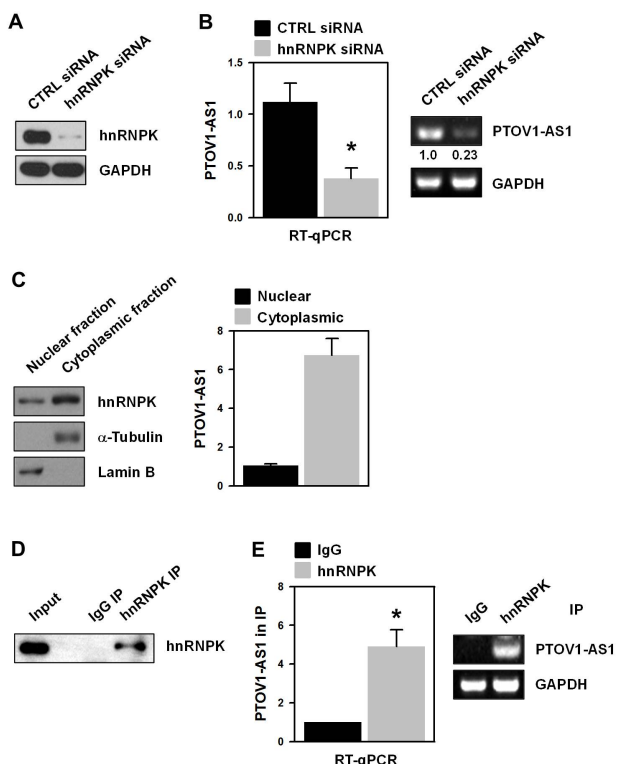

Fig. 1. Knockdown of hnRNPK decreases the expression of PTOV1-AS1. (A, B) To verify RNA sequencing data, HeLa cells were transfected with control (CTRL) or hnRNPK siRNA. The level of hnRNPK was assessed by Western blotting (A), and the level of PTOV1-AS1 was determined by RT-qPCR and semi-quantitative PCR (B: left and right panels, respectively). (C) Cellular fractionation was performed as described in the Materials and Methods section. The levels of $\alpha$-tubulin and lamin B were checked as cytoplasmic and nuclear markers, respectively. Localization of PTOV1-AS1 was determined by RT-qPCR. (D, E) Interaction of PTOV1-AS1 with hnRNPK was assessed by RNP IP as described in the Materials and Methods section. To verify efficient hnRNPK IP, Western blotting was performed with IP materials (D). The level of PTOV1-AS1 in the hnRNPK IP materials was determined by RT-qPCR and semi-quantitative PCR (E: left and right panel, respectively). right panel). Ribonucleoprotein immunoprecipitation (IP) with an hnRNPK antibody (Fig. 1D) indicated that hnRNPK can interact with PTOV1-AS1 (Fig. 1E). These results suggest that hnRNPK influences the expression of its target genes via PTOV1-AS1.

PTOV1-AS1 functions as a molecular decoy for miR-1207-5p Several prediction programs were used to identify miRNA recognition motifs in PTOV1-AS1 transcripts. Since PTOV1AS1 has five binding sites for miR-1207-5p, we hypothesized that hnRNPK can regulate the functions of miR-1207-5p via PTOV1-AS1 (Fig. 2A). First, we evaluated the enrichment of PTOV1-AS1 in Ago2 IP (Fig. 2B). As shown in Fig. 2C, PTOV1-AS1 was highly enriched in Ago2 IP, indicating that it is closely associated with miRISC. Therefore, we compared and analyzed predicted miR-1207-5p target genes (TargetScan7.0) and RNA sequencing data (Fig. 2D). In total, 97 miR-1207-5p target genes were downregulated in hnRNPK-silenced cells (Suppl. Fig. S1). We also checked the levels of several genes in PTOV1-AS1-silenced cells and chose heme oxygenase-1 (HO-1) for further studies. Based on our results, it was hypothesized that hnRNPK regulates PTOV1-AS1, which acts as a decoy for miR-1207-5p. To evaluate our hypothesis, several points need to be confirmed. First, we checked whether hnRNPK directly
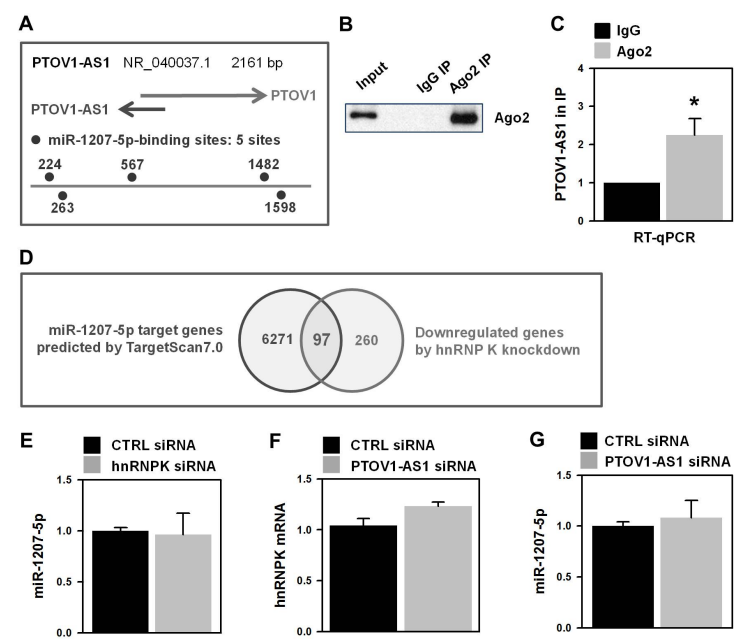

Fig. 2. PTOV1-AS1 acts as a putative endogenous decoy for miR-1207-5p. (A) Schematic diagram shows that PTOV1-AS1 has five binding sites for miR-1207-5p. (B, C) To check whether PTOV1-AS1 is associated with miRNA-mediated RISC, Ago IP was performed (B) and the level of PTOV1-AS1 was assessed by RT-qPCR (C). (D) To identify common genes regulated by hnRNPK and miR-1207-5p, we compared and analyzed gene lists. In total, 97 genes were identified as putative target genes of PTOV1-AS1. (E) To rule out the possibility that hnRNPK regulates miRNA biogenesis, the level of miR-1207-5p was determined by RT-qPCR in hnRNPK-silenced cells. $(\mathrm{F}, \mathrm{G})$ HeLa cells were transfected with control (CTRL) or PTOV1-AS1 siRNA, and the levels of hnRNPK and miR-1207-5p were determined by RT-qPCR. 
influences miR-1207-5p expression. The expression level of miR-1207-5p in hnRNPK-silenced cells was almost identical to that in control cells (Fig. 2E). Next, the effects of PTOV1-AS1 silencing on the expression of hnRNPK and miR-1207-5p were examined. Neither hnRNPK nor miR-1207-5p was affected by PTOV1-AS1 expression (Figs. 2F and 2G, respectively).

\section{The hnRNPK/PTOV1-AS1/miR-1207-5p axis is implicated in HO-1 expression}

Based on the above results, we found that hnRNPK regulates the level of PTOV1-AS1 which has five miR-1207-5p binding sites. Therefore, we investigated whether miR-1207-5p increases the enrichment of PTOV1-AS1 in Ago2 IP (Fig. 3A). The interaction of PTOV1-AS1 with miRISC increased in response to the overexpression of miR-1207-5p. Moreover, miR-1207-5p suppressed the expression of $\mathrm{HO}-1$ at the mRNA and protein levels (Fig. 3B), indicating that miR-1207-5p is a novel HO-1targeting miRNA. Next, we examined the effect of hnRNPK silencing on HO-1 expression. The knockdown of hnRNPK increased the level of HO-1 mRNA in Ago2 IP (Fig. 3C) and decreased the mRNA and protein expression of HO-1 (Fig. 3D). Similar to the results obtained for hnRNPK, we observed that PTOV1-AS1-silenced cells showed highly enriched HO-1 mRNA following Ago2 IP (Fig. 3E), and the knockdown of PTOV1-AS1 also decreased the levels of HO-1 at the mRNA and protein levels (Fig. 3F). In the absence of hnRNPK and
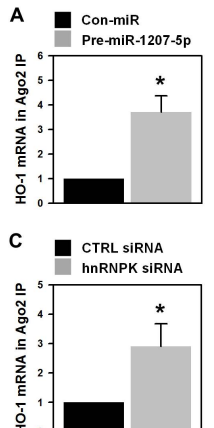

E CTRL SIRNA

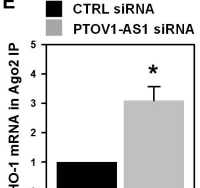

B

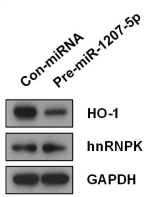

D

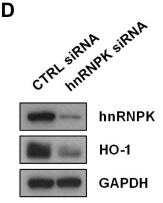

$\mathbf{F}$

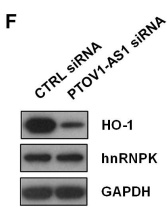

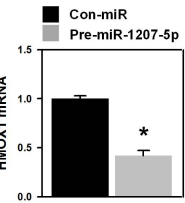

CTRL siRnA

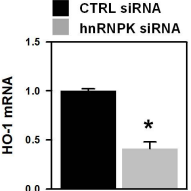

ctRL siRna

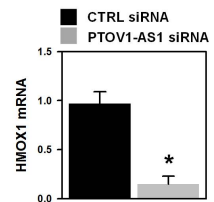

Fig. 3. The hnRNPK/PTOV1-AS1/miR-1207-5p axis is implicated in the expression of HO-1. To examine the effects of miR-1207-5p, hnRNPK, and PTOV1-AS1 on HO-1 expression, HeLa cells were transfected with miR-1207-5p mimic (A, B), siRNA targeting hnRNPK (C, D), or PTOV1-AS1 (E, F). The enrichment of HO-1 mRNA in Ago2 IP was assessed by RT-qPCR in transfected cells (A, C, E). The protein and mRNA expression levels of $\mathrm{HO}-1$ were determined by western blotting and RT-qPCR, respectively (B, D, F). Antibodies and primers used for these experiments are provided in Supplementary Table 2 and 3, respectively.
PTOV1-AS1, miR-1207-5p easily accesses the 3' UTR of HO-1 mRNA, resulting in the downregulation of HO-1. Taken together, these results demonstrated that hnRNPK regulates the expression of HO-1 via the miR-1207-5p decoying PTOV1-AS1.

\section{The hnRNPK/PTOV1-AS1/miR-1207-5p/HO-1 axis is closely associated with proliferation}

Since HO-1 is closely associated with the proliferation of cancer cells, we investigated the role of the hnRNPK/PTOV1/ miR-1207-5p/HO-1 axis in cell proliferation. Accordingly, cells were transfected with hnRNPK siRNA (Fig. 4A, B), PTOV1-AS1 siRNA (Fig. 4C, D), pre-miR-1207-5p (Fig. 4E, F), or HO-1 siRNA (Fig. 4G, H). Viable cells were quantified by an MTS cell proliferation assay after incubation for $48 \mathrm{~h}$. As expected, all transfected cells showed decreased proliferation compared to control cells (Figs. 4A, C, E, and G). The clonogenic ability of cells indicates survival and proliferation. Therefore, we tested the clonogenic abilities of transfected cells by a colony forming assay. Similar to the results of the MTS assay, decreased colony numbers were observed in all transfected cells (Figs. 4B, D, F, and H).

In summary, our results demonstrate that the knockdown of hnRNPK potentiates the inhibitory function of miR-1207-5p by downregulating miR-1207-5p decoying transcripts, i.e., PTOV1-AS1, resulting in decreased HO-1 expression. Moreover, the down-regulation of $\mathrm{HO}-1$ inhibits proliferation and clonogenic ability of cells.

\section{DISCUSSION}

miRNAs are closely associated with multiple oncogenic
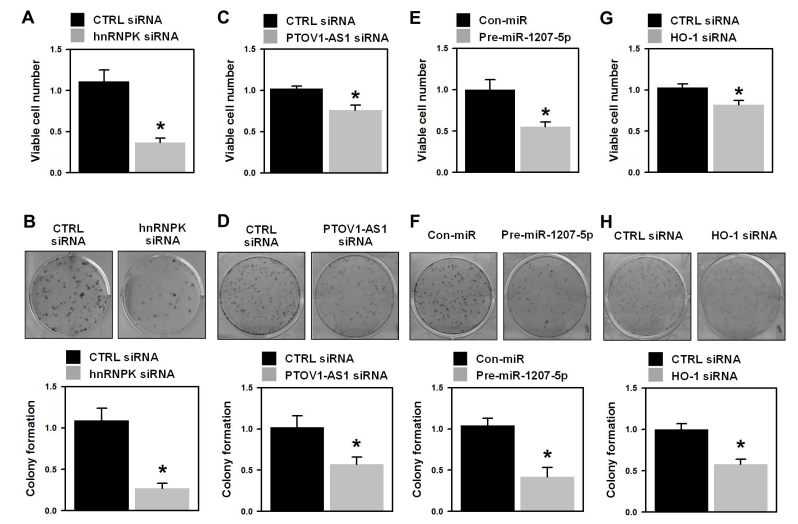

Fig. 4. HO-1 is involved in cell proliferation and clonogenicity. HeLa cells were transfected with hnRNPK siRNA (A, B), PTOV1-AS1 siRNA (C, D), pre-miR-1207-5p (E, F), or HO-1 siRNA $(G, H)$. After $24 \mathrm{~h}$ posttransfection, cells were resuspended into 96-well plates for the MTS assay $(A, C, E$, and $G$ ) or in 6-well plates for the colony forming assay $(B, D, F$, and $H)$. Cell proliferation and clonogenicity were determined as described in the Materials and Methods section. 
processes, including proliferation, migration, invasion, and angiogenesis. In general, miRNAs induce the decay or translational suppression of target mRNAs via MREs in their $3^{\prime}$ UTRs. The 3' UTRs of protein-coding transcripts are closely associated with cancer progression by post-transcriptional gene regulation. Alteration of the $3^{\prime}$ UTR may influence not only the stability or translation of its transcripts in cis, but also miRNA functions by sharing MREs in trans (10). Recently, ceRNAs have been identified as a new class of functional RNAs that act as miRNA sponges (11). ceRNAs, including several non-coding RNAs, such as pseudogenes, antisense long ncRNAs (IncRNAs), and circular RNAs, compete with target mRNAs to provide MREs to corresponding miRNAs, suggesting that they are essential for the control of the quantity of miRNAs. For example, antisense transcripts for the $\beta$-APP-cleaving enzyme 1 gene (BACE1) hinder the miR-485-5p binding site of its sense transcript, which consequently inhibits miRNA decay and the translational suppression of BACE1 (12). HOTAIR (HOX antisense intergenic RNA) is a well-studied antisense IncRNA transcribed from the $\mathrm{HOHC}$ locus from an intergenic position and in an antisense direction to the flanking HOXC11 and HOXC12 loci (13). It plays a functional role in oncogenic processes, influencing proliferation, mobility, invasion, and metastasis.

Prostate tumor overexpressed gene 1 (PTOV1) is overexpressed in the early and late stages of prostate cancer (14). PTOV1 was initially identified in prostate cancer, but it has recently been reported to enhance the population of cancer stem cells via the activation of $\mathrm{Wnt} / \beta$-catenin signaling in breast cancer (15). PTOV1 is also a key player in tumor progression and is associated with a poor prognosis in laryngeal squamous cell carcinoma, urothelial carcinoma, and ovarian cancer (16-18). In addition to the oncogenic roles of its sense transcripts, our findings strongly suggest that antisense PTOV1-AS1 is also involved in cancer proliferation via miR-1207-5p. We found that PTOV1-AS1 can bind to and inhibit the function of miR-1207-5p. The knockdown of PTOV1-AS1 did not influence the levels of hnRNPK and miR-1207-5p but decreased the expression of $\mathrm{HO}-1$ by enhancing the enrichment of its mRNA in miRISC.

HO-1 is highly expressed in various types of cancer, and its expression level is positively correlated with cancer progression and poor prognosis in patients (19). It is also highly expressed in tumor-associated macrophages, suggesting that $\mathrm{HO}-1$ plays an important role in cancer microenvironments (20). Since cancer cells have high metabolic rates that enable rapid proliferation, they exhibit extremely high oxidative stress. Low expression of HO-1 exacerbates reactive oxygen species-induced DNA damage and accelerates the initiation of carcinogenesis. Also, high levels of HO-1 are associated with rapid proliferation, invasiveness, and drug resistance (21-26). In response to various stimuli, such as growth factors and cytokines released from stromal cells, cancer cells express high levels of HO-1 via the activation of several transcriptional factors, including nuclear factor erythroid 2-related factor 2 (Nrf2), nuclear factor kappaB (NF-kB), and activator protein 2 (AP2) (27). In addition to transcriptional regulation, the expression level of HO-1 is also governed by a post-transcriptional mechanism. Suppression of HO-1 by miR-378 was reported to inhibit the proliferation and metastasis of lung carcinoma (28). In renal cell carcinoma, the downregulation of $\mathrm{HO}-1$ by miR-200c makes cancer cells more susceptible to chemotherapeutic agents (24). In this report, we identified a novel HO-1-targeting miRNA, miR-1207-5p, involved in the inhibition of the proliferation and clonogenicity of HeLa cells. We also found that hnRNPK-regulated PTOV1-AS1 acts as a molecular decoy for miR-1207-5p.

\section{MATERIAIS AND METHODS}

\section{Cell culture and transfection}

HeLa cervical carcinoma cells were cultured at $37^{\circ} \mathrm{C}$, and $5 \%$ $\mathrm{CO}_{2}$ in Dulbecco's modified Eagle's medium (Hyclone, Logan, UT, USA) supplemented with $10 \%$ fetal bovine serum and a $1 \%$ antibiotic-antimycotic solution (GIBCO-BRL, Grand Island, NY, USA). For transient transfection of siRNA or miRNA, cells were plated at an appropriate stage of confluency and transfected using Lipofectamine 2000 (Invitrogen, Carlsbad, CA, USA) according to the manufacturer's protocol. A mixture of two different siRNAs targeting the coding region or 3' UTR was used to silence hnRNPK. To reduce the level of PTOV1-AS1, specific siRNA was designed and synthesized (Bioneer, Daejeon, South Korea). The sequences of these siRNAs are provided in Supplementary Table 1.

\section{Westem blot analysis}

For western blot analyses, cells were rinsed with ice-cold phosphate-buffered saline (PBS) and lysed using radioimmunoprecipitation (RIPA) buffer containing protease inhibitors and phosphatase inhibitors (Roche, Basel, Switzerland) (10). Cell lysates were clarified by centrifugation, and the protein concentration was quantified using Bradford reagent (Bio-Rad Laboratories, Hercules, CA, USA). Equal amounts of cell lysate were separated by SDS-polyacrylamide gel electrophoresis and then transferred to polyvinylidene difluoride membranes (Millipore, Billerica, MA, USA). Membranes were blocked with 5\% skim milk (BD Biosciences, San Jose, CA, USA) for 1 hour at room temperature. After that, the membranes were incubated overnight at $4^{\circ} \mathrm{C}$ with the indicated primary antibodies, washed with Tris-buffered saline and Tween 20 (TBST), and then incubated with the appropriate secondary antibodies for 1 hour at room temperature. Antibodies used for this study are provided in Supplementary Table 2.

\section{Real-time quantitative polymerase chain reaction (RT-qPCR) analysis}

Total RNA was isolated using TRIzol reagent (Invitrogen, Carlsbad, CA, USA) according to the manufacturer's instructions 
and used as a template to synthesize complementary DNA (cDNA) using the SuperScript III First-Strand Synthesis System (Invitrogen, Carlsbad, CA, USA). The mRNA level was quantified by RT-qPCR (ABI Prism 7900) using power SYBR ${ }^{\circledR}$ Green PCR Master Mix (Applied Biosystems, Foster City, CA, USA). The primers used in this study are provided in Supplementary Table 3. The level of miRNA was assessed using a miR-1207-5p-specific TaqMan primer (Applied Biosystems, Foster City, CA, USA).

\section{Ribonucleoprotein immunoprecipitation (RNP-IP)}

For RNP-IP, Dynabeads ${ }^{\mathbb{R}}$ Protein G (Thermo Fisher Scientific, Rockford, IL, USA) were coated with control IgG (Santa Cruz Biotechnologies, Santa Cruz, CA, USA) or hnRNP K antibody (Abcam, Cambridge, UK). For Ago2 IP, beads were coated with an Ago2 antibody (Sigma, St. Louis, MO, USA). Cytoplasmic lysates were prepared using PEB lysis buffer containing a protease inhibitor, a phosphatase inhibitor, and RNaseOUT (Invitrogen, Carlsbad, CA, USA). Equal amounts of lysates were incubated with antibody-coated Dynabeads for 4 hours at $4^{\circ} \mathrm{C}$. hnRNP K-IP materials were washed several times with PEB buffer and treated with DNase I (Ambion, Austin, TX, USA) and protease K (Bioneer, Daejeon, South Korea). The RNA was isolated with acid phenol (Ambion) and precipitated with absolute ethanol overnight at $-20^{\circ} \mathrm{C}$. The mRNA expression level was determined by RT-qPCR, as described above.

\section{Subcellular fractionation}

For isolation of the cytosolic and nuclear fraction, cells were harvested and lysed with digitonin-containing RSB buffer. After centrifugation at $2,000 \times \mathrm{g}$ for $10 \mathrm{~min}$, the supernatant was transferred to a new tube (Cytosolic extracts). The remaining pellet was washed five times with RSB buffer and lysed with RIAP buffer. Nuclear extracts were isolated by centrifugation at 13,200 rpm for 20 min.

\section{Determination of cell proliferation and clonogenicity}

Transfected cells were suspended in 96-well plates $\left(1 \times 10^{4}\right.$ cells per well), and cells were then stained after 48 hours with MTS solution containing PMS according to the MTS Assay Kit protocol (Promega, Madison, WI, USA). A colony-forming assay was performed to determine clonogenic ability,. Briefly, transfected cells were seeded in triplicate in 6-well plates $(3 \times$ $10^{2}$ cells per well) and incubated at $37^{\circ} \mathrm{C}$ and $5 \% \mathrm{CO}_{2}$ in complete DMEM medium for 2 wks. After incubation, cells were washed in cold PBS and fixed with $4 \%$ paraformaldehyde (Biosesang, Gyeonggi, South Korea) for 5 min, then washed twice with cold PBS. Fixed cells were stained with $0.2 \%$ crystal violet for 1 hour and washed 3 times with cold PBS, and then stained colonies were counted.

\section{ACKNOWLEDGEMENTS}

This research was supported by the Sungkyun Research Fund,
Sungkyunkwan University, 2016 [S-2016-2280-000].

\section{CONFLICTS OF INTEREST}

The authors have no conflicting financial interests.

\section{REFERENCES}

1. Katayama S, Tomaru Y, Kasukawa T et al (2005) Antisense transcription in the mammalian transcriptome. Science 309, 1564-1566

2. Ozsolak F, Kapranov P, Foissac S et al (2010) Comprehensive polyadenylation site maps in yeast and human reveal pervasive alternative polyadenylation. Cell 143, 1018-1029

3. Villegas VE and Zaphiropoulos PG (2015) Neighboring gene regulation by antisense long non-coding RNAs. Int J Mol Sci 16, 3251-3266

4. Bartel DP (2009) MicroRNAs: target recognition and regulatory functions. Cell 136, 215-233

5. Salmena L, Poliseno L, Tay $Y$, Kats L and Pandolfi PP (2011) A ceRNA hypothesis: the Rosetta Stone of a hidden RNA language? Cell 146, 353-358

6. Cesana M and Daley GQ (2013) Deciphering the rules of ceRNA networks. Proc Natl Acad Sci U S A 110, $7112-$ 113

7. Maines MD (1988) Heme oxygenase: function, multiplicity, regulatory mechanisms, and clinical applications. FASEB J 2, 2557-2568

8. Ali F, Zakkar M, Karu K et al (2009) Induction of the cytoprotective enzyme heme oxygenase- 1 by statins is enhanced in vascular endothelium exposed to laminar shear stress and impaired by disturbed flow. J Biol Chem 284, 18882-18892

9. Dulak J, Loboda A, Zagorska A and Jozkowicz A (2004) Complex role of heme oxygenase-1 in angiogenesis. Antioxid Redox Signal 6, 858-866

10. Karreth FA and Pandolfi PP (2013) ceRNA cross-talk in cancer: when ce-bling rivalries go awry. Cancer Discov 3, 1113-1121

11. Kartha RV and Subramanian S (2014) Competing endogenous RNAs (ceRNAs): new entrants to the intricacies of gene regulation. Front Genet 5, 8

12. Faghihi MA, Zhang M, Huang J et al (2010) Evidence for natural antisense transcript-mediated inhibition of microRNA function. Genome Biol 11, R56

13. Rinn JL, Kertesz M, Wang JK et al (2007) Functional demarcation of active and silent chromatin domains in human HOX loci by noncoding RNAs. Cell 129, 1311-1323

14. Benedit P, Paciucci R, Thomson TM et al (2001) PTOV1, a novel protein overexpressed in prostate cancer containing a new class of protein homology blocks. Oncogene 20, 1455-1464

15. Cui Y, Ma W, Lei F et al (2016) Prostate tumour overexpressed-1 promotes tumourigenicity in human breast cancer via activation of $\mathrm{Wnt} /$ beta-catenin signalling. J Pathol 239, 297-308

16. Yang L, Wang H, Wang $\mathrm{Y}$ et al (2016) Prostate tumor 
overexpressed-1, in conjunction with human papillomavirus status, predicts outcome in early-stage human laryngeal squamous cell carcinoma. Oncotarget 7, 31878-31891

17. Rausch S, Hennenlotter J, Scharpf M et al (2016) Prostate tumor overexpressed 1 expression in invasive urothelial carcinoma. J Cancer Res Clin Oncol 142, 937-947

18. Guo F, Feng L, Hu JL et al (2015) Increased PTOV1 expression is related to poor prognosis in epithelial ovarian cancer. Tumour Biol 36, 453-458

19. Chau LY (2015) Heme oxygenase-1: emerging target of cancer therapy. J Biomed Sci 22, 22

20. Nishie A, Ono M, Shono T et al (1999) Macrophage infiltration and heme oxygenase-1 expression correlate with angiogenesis in human gliomas. Clin Cancer Res 5, 1107-1113

21. Chen GG, Liu ZM, Vlantis AC, Tse GM, Leung BC and van Hasselt CA (2004) Heme oxygenase-1 protects against apoptosis induced by tumor necrosis factor-alpha and cycloheximide in papillary thyroid carcinoma cells. J Cell Biochem 92, 1246-1256

22. Liu ZM, Chen GG, Ng EK, Leung WK, Sung JJ and Chung SC (2004) Upregulation of heme oxygenase- 1 and p21 confers resistance to apoptosis in human gastric cancer cells. Oncogene 23, 503-513

23. Mayerhofer M, Florian S, Krauth MT et al (2004)
Identification of heme oxygenase- 1 as a novel BCR/ABLependent survival factor in chronic myeloid leukemia. Cancer Res 64, 3148-3154

24. Gao C, Peng FH and Peng LK (2014) MiR-200c sensitizes clear-cell renal cell carcinoma cells to sorafenib and imatinib by targeting heme oxygenase-1. Neoplasma 61 , 680-689

25. Furfaro AL, Piras $S$, Passalacqua $M$ et al (2014) HO-1 up-regulation: a key point in high-risk neuroblastoma resistance to bortezomib. Biochim Biophys Acta 1842, 613-622

26. Kongpetch S, Kukongviriyapan V, Prawan A, Senggunprai L, Kukongviriyapan U and Buranrat B (2012) Crucial role of heme oxygenase- 1 on the sensitivity of cholangiocarcinoma cells to chemotherapeutic agents. PLoS One 7, e34994

27. Lavrovsky Y, Schwartzman ML, Levere RD, Kappas A and Abraham NG (1994) Identification of binding sites for transcription factors NF-kappa B and AP-2 in the promoter region of the human heme oxygenase 1 gene. Proc Natl Acad Sci U S A 91, 5987-5991

28. Skrzypek K, Tertil M, Golda S et al (2013) Interplay between heme oxygenase-1 and miR-378 affects nonmall cell lung carcinoma growth, vascularization, and metastasis. Antioxid Redox Signal 19, 644-660 